\title{
La malédiction, l'honneur et la spéculation
}

Principes historiques de la propriété foncière en Afrique de l'Ouest

Jacky Bouju

\section{(2) OpenEdition}

\section{Journals}

Édition électronique

URL : http://journals.openedition.org/apad/4006

DOI : $10.4000 /$ apad.4006

ISSN : 1950-6929

Éditeur

LIT Verlag

\section{Édition imprimée}

Date de publication : 15 juin 2009

Pagination : 71-91

\section{Référence électronique}

Jacky Bouju, « La malédiction, l'honneur et la spéculation », Bulletin de l'APAD [En ligne], 29-30 | 2009,

mis en ligne le 16 juin 2010, consulté le 08 septembre 2020. URL : http://journals.openedition.org/ apad/4006 ; DOI : https://doi.org/10.4000/apad.4006

Ce document a été généré automatiquement le 8 septembre 2020.

Bulletin de I'APAD 


\section{La malédiction, l'honneur et la spéculation}

Principes historiques de la propriété foncière en Afrique de l'Ouest

Jacky Bouju

1 En Afrique de l'Ouest, la marchandisation rapide des terres et le développement de conflits fonciers très violents, tant urbains que ruraux, invitent à réinterroger à la lumière des données ethnographiques abondantes dont on dispose aujourd'hui, la vieille question des fondements non marchands de la possession des ressources naturelles et de la terre en particulier dans les sociétés ouest africaines. Le but n'est pas, bien évidemment, de cautionner une quelconque vision mythique d'un état « naturel » du rapport ancien des populations à leur environnement ou de donner dans la nostalgie d'un âge d'or des « droits traditionnels » qui serait révolu.

Dans nombre de pays africains, l'accès à la terre, que ce soit pour un usage d'habitation, de culture ou de pâture ou pour la spéculation foncière, est devenu aujourd'hui un enjeu majeur pour tous qui s'accompagne, partout, de grandes violences tant physiques que symboliques. Il s'agira donc ici de prendre un peu de recul pour comprendre ce qui est en train de se jouer en profondeur dans l'espace social apparemment anomique des transactions foncières urbaines et rurales contemporaines. Pour ce faire, nous essaierons d'apporter des éléments de réponse aux questions suivantes: quelles représentations de leurs maîtrises foncières se font les acteurs des transactions contemporaines? Quels sont les répertoires normatifs qu'ils mobilisent à ces occasions, que ce soit pour s'y arc-bouter, les détourner ou les contester vigoureusement? Quelles conceptions aussi se font-ils du droit foncier de l'Autre?

\section{La possession « traditionnelle » comme problème théorique}

3 Il convient avant tout de préciser le sens des concepts utilisés car les confusions en ce domaine furent à l'origine de nombreux malentendus dont témoigne la vigueur des débats anciens sur la nature du Droit dans les sociétés "primitives», ou ceux plus 
modernes, sur la nature collective, commune ou privée de la "propriété » des ressources dans les sociétés " traditionnelles » (Cohen-Tanuggi, 1985; Verdier, 1986; Le Bris et al., 1983; Madjarian, 1991; Schlager et Ostrom, 1992 ; Bouju, 2004b).

Dans la culture euro-occidentale, l'appropriation. renvoie à des règles explicites d'assignation d'une ressource à un usager (un sujet juridique) ou à un usage, et à la définition des maîtrises d'usage et de transfert qui en découlent. Cette conception de la propriété qui est aujourd'hui mondialisée a une histoire ancienne qui commence avec l'apparition de l'État et l'invention de l'écriture (Handman, 1991 ; Lamaison, 1991). Elle apparaît à la conjonction de deux processus historiques à la fois symboliques et politiques : d'une part, le détachement des richesses par rapport aux individus qui les produisent et d'autre part, celui de la séparation de la personne comme individu distinct de son appartenance à un collectif communautaire. À partir de là, on assiste à une élaboration juridique progressive du concept européen de propriété ${ }^{2}$ qui n'acquiert son caractère privé absolu qu'avec la généralisation de la marchandise et l'individualisation du sujet de droit (Handman, citant Karl Marx, 1991 : 606).

5 Contrairement à cette conception juridique de la propriété qui considère toujours l'appropriation comme un rapport simple et direct établit entre un individu (ou une personne morale) et une ressource, l'anthropologique économique a, très tôt, considéré la possession comme un rapport social historiquement établi entre des acteurs sociauxindividuels ou collectifs par le truchement du contrôle exclusif d'une ressource. Il reste cependant que l'usage de la notion de "possession", de "propriété » ou de « Droit » par les anthropologues est resté lourd d'ambiguïtés que les meilleurs travaux d'anthropologie économique n'ont jamais réussi à lever complètement ${ }^{3}$. Il faudra attendre les années 1990 pour assister à un renouvellement de la question grâce à la théorie des maîtrises foncières développée par Schlager et Ostrom (1992), précisée par Sandberg (1994) et enfin, reprise et mise en forme en France par Etienne Le Roy (Le Roy, 1996, 1998). Cette nouvelle théorie a permis aux anthropologues d'éviter l'usage incertain de la notion de " propriété » dans la description et l'analyse des formes prémarchandes de possession des ressources naturelles.

\section{La notion de maîtrise foncière}

6 La théorie des maîtrises foncières décompose la notion de possession d'une ressource en ses différentes dimensions. Pour la terre, celles-ci renvoient à deux niveaux de "maîtrise " de la ressource foncière : la «maîtrise de l'usage " (de la terre) et la «maîtrise du contrôle " (de l'usage de la terre). La maitrise de l'usage se décompose à son tour en deux maîtrises principales : «l'accès à la ressource » (qui est la plus faible maîtrise qu'on puisse détenir) et « le prélèvement de la ressource » (partout en Afrique l'appartenance lignagère conférait automatiquement la maîtrise de l'accès et de l'exploitation du domaine patrimonial).

7 Ensuite, au niveau supérieur, on a la « maîtrise du contrôle de l'accès à la ressource ». Celle-ci se décompose aussi en deux maîtrises : la « gestion de la ressource » (la capacité d'autoriser et d'organiser l'accès et le prélèvement) et enfin « le pouvoir d'exclure » (la capacité d'interdire l'accès et le prélèvement), pouvoir éminent qui était détenu par des «maîtres de la terre » ou des « chefs de terre ». Ces quatre maîtrises ${ }^{4}$, ordonnées de la plus faible à la plus forte, permettent de décrire précisément les formes locales d'usage 
et de possession effective d'une ressource. Dans mes travaux antérieurs, j'ai repris à mon compte et adapté cette approche qui permet de savoir précisément de quoi les gens parlent en décrivant leurs « droits » fonciers (Bouju, 1998b ; Bouju et al., 2004).

8 Toujours et partout en Afrique de l'Ouest, c'était le premier défrichage d'une brousse qui constituait l'acte fondamental de prise de possession de l'espace par le fondateur. Cet acte engendrait une maitrise exclusive (permanente et inaliénable) de la ressource foncière. Mais, l'acte de défrichage ne suffisait pas à établir la possession coutumière. Celle-ci n'était validée, en quelques sortes, que par la conclusion d'un pacte d'alliance avec les divinités chtoniennes (génies d'eau, génies de brousse) garantes de la fertilité de la nature qui, partout, étaient considérées comme les «vrais » propriétaires des lieux. Ce pacte rituel avait avant tout valeur juridique. En effet, l'ensemble du dispositif rituel associé à l'exploitation annuelle du milieu ${ }^{5}$. garantissait la préséance du clan issu des descendants du défricheur vis-à-vis des tiers et instaurait leur maitrise exclusive sur le terroir soumis à l'influence de ces divinités chtoniennes. Ainsi, dans la coutume Dogon, la maîtrise de l'accès et du prélèvement de la ressource, le "droit de culture ", était clairement distincte de la maîtrise du contrôle de l'accès et du pouvoir d'exclure. Le seul pouvoir que détenaient les descendants des fondateurs (qu'on qualifie aujourd'hui d'autochtones) était le monopole de la relation rituelle aux divinités chtoniennes. Selon les conceptions locales, ces divinités chtoniennes contrôlaient la fertilité et l'aridité de la nature mais aussi la fécondité des femmes et seuls les autochtones pouvaient les manipuler. C'est par le truchement de ce pacte fondateur de leur monopole rituel que les autochtones disposaient du pouvoir éminent d'exclure. Le pacte attestait la préséance territoriale des autochtones qui, traditionnellement, constituait un véritable pouvoir local.

Chez les Dogon, la maîtrise d'usage de l'espace découlait de l'appartenance à un domaine patrimonial constitué d'un quartier-lignage, la "grande maison ", gin'na, et de ses terres, localisés dans un village abritant éventuellement plusieurs domaines de ce type. C'était donc les membres d'une grande maison qui détenaient une maitrise inaliénable d'exploitation directe et indépendante de la portion du terroir qui avait été défrichée par leur ancêtre fondateur. Traditionnellement donc, la maîtrise d'usage de l'espace était acquise par la naissance et elle s'étendait avec l'accroissement du nombre des descendants et l'extension concomitante des surfaces cultivées. Toutefois, la fertilité du terroir restait sous le contrôle rituel du lignage autochtone qui en avait la maîtrise exclusive. C'est à ce niveau que l'interdiction de planter des arbres ou d'en utiliser les fruits (un marqueur très répandu signalant l'existence d'une maitrise exclusive de la ressource) prenait toute sa signification foncière.

10 La maîtrise de la gestion du terroir comportait comme prérogative la possibilité de prêter une parcelle de culture à un parent, un allié, un ami ou un voisin. Ce " prêt de terre " était compris comme un transfert d'usage temporaire (même s'il durait plusieurs générations), une sorte de délégation intransmissible et parfois conditionnelle. Tout chef de grande maison était ainsi libre de transférer sa maîtrise d'usage en prêtant une parcelle de terre à un cultivateur «étranger » mais, en tant que «tuteur », il conservait la maîtrise de sa gestion. Mais quand le tutorat se reproduisait pendant plusieurs générations, la maîtrise d'usage délégué devenait instable et lourde d'ambiguïtés. Aujourd'hui, la crise générale du tutorat se manifeste par une restriction du contenu et de la durée des maîtrises d'usage temporaires accordées aux cultivateurs immigrants. Cette organisation foncière rurale relève d'une logique patrimoniale ${ }^{6}$ qui, 
par son extension géographique, apparaît comme une caractéristique du développement historique du peuplement agraire ouest africain.

\section{La transmission des maîtrises foncières ou la métamorphose de la propriété individuelle en propriété commune}

11 Une parcelle de culture était d'abord défrichée individuellement, puis cultivée collectivement par le défricheur et ses fils. Ensuite, au décès du défricheur, la parcelle devenait un patrimoine commun hérité par tous ses descendants en ligne masculine. La coutume dogon décrit la possession d'une richesse (personne, terre ou chose) en disant que la richesse "a échu » à cette personne. Maintenant, elle est " pour lui », wo mõ, ce qui signifie que cette personne est le gardien de cette richesse; au nom du groupe des ayant-droits, il a la "mainmise? "., numó mõ, sur la chose possédée. Après quelques générations la parcelle, augmentée à chaque nouvelle génération de quelques défrichages supplémentaires, devenait le terroir domanial de la grande maison. C'est donc la pratique effective des règles de transmission qui instituait la continuité de la grande maison comme «grande famille» et comme domaine patrimonial. C'est au niveau de l'héritage («ce qui était passé aux générations suivantes ») et de la répartition de " ce qui échoit » et que "l'on ramasse » que se joue, en partie, la continuité des rapports sociaux de production traditionnels.

12 Ainsi, au plan coutumier, la transmission par héritage et succession en ligne patrilinéaire conférait à toute richesse transmise les qualités d'indivisibilité et d'inaliénabilité, enclenchant de ce fait, et ce dès la première transmission, un processus de patrimonialisation constitutif à terme d'une future "grande maison» (Bouju, 1984). Dans ces sociétés, un homme adulte jouissait donc, simultanément, de la gestion exclusive de ses propriétés personnelles (appropriées par son activité prédatrice ou son travail de défrichement) et de l'accès exclusif au patrimoine commun.

Le patrimoine reçu et possédé par héritage demeurait fortement attaché à l'identité de la grande maison dont il relevait et à la personne du «maitre » qui en était le gardien au nom de tous. Les règles de transmission constituaient le domaine patrimonial en un champ d'appropriation et de répartition exclusive des richesses encloses. Les frontières de la transmission excluent toujours ceux qui se trouvent au-delà (les femmes, les membres des autres grandes maisons, les gens de caste, les étrangers, etc.) donnant ainsi à voir la totalité du groupe communautaire ayant droit d'accès au patrimoine commun et à concevoir son identité comme groupe de transmission. Indicateur précieux des limites pratiques de la famille étendue, l'héritage est extrêmement sensible à l'évolution des valeurs et aux changements économiques et religieux et, aujourd'hui, selon les lieux et les circonstances, ces règles dogon anciennes sont très diversement respectées. Ce processus de métamorphose d'une possession initialement individuelle en propriété commune par la transmission à la descendance concernait toutes les richesses possibles.

Le "trésor " patrimonial (Weiner, 1988; Godelier, 1997) constitué par les personnes (enfants, épouses, esclaves, cadets et clients dépendants) les choses (traditions et secrets de famille) et les biens (bétail, argent, armes, etc) détenus par la grande maison ne concerne pas seulement les ressources matérielles. Il comprenait aussi les ressources symboliques (cultes, autels, interdits, etc.), les ressources organisationnelles (normes et règles coutumières), les ressources cognitives (les traditions historiques et 
mythiques) et les ressources socio-politiques (liens d'alliance, parenté à plaisanterie). La sphère de transmission du "trésor " patrimonial construisait les limites de l'identité communautaire. $\mathrm{Du}$ point de vue socioanthropologique, ce processus transgénérationnel doit être mis en regard du fait que tout bien acquis, produit, obtenu de quelconque manière par un cadet ou un dépendant devait être immédiatement remis au père/aîné, chef famille, maître de maison, qui pouvait en droit le garder pour lui-même, le donner à autrui ou le rendre à son premier propriétaire (au sens marchand du terme). C'est donc le chef de grande famille, le maître, qui était le gardien de la maîtrise exclusive des richesses d'une grande maison. Mais que signifiait la maitrise exclusive d'une maison sur son domaine foncier?

Dans notre culture, la propriété est individuelle et signifie le droit pour un sujet d'user, jouir et disposer de manière exclusive et absolue d'un objet qui est distinct de luimême. Mais, ainsi qu'on vient de le voir, dans la coutume Dogon c'est plus compliqué. En général, la maitrise commune du patrimoine foncier était inséparable de l'identité de la grande maison et de ses membres. Et il en allait de même pour les autres richesses possédées. En l'occurrence, ce sont ces possessions non marchandes, ces biens communs, qui devraient être qualifiés de "propriétés»! En effet, dans ce contexte historique de la maîtrise exclusive de richesses patrimoniales, les choses possédées n'étaient pas séparables de leur possesseur et ce faisant, elles participaient fondamentalement de l'identité individuelle et collective des personnes et des groupes. Ce qui correspond bien à l'autre définition de la "propriété", à savoir être une caractéristique propre à une entité qui permet de l'identifier et de la définir !

\section{La sécurisation coutumière des maîtrises foncières}

Cette conception générale de la propriété est attestée dans la plupart des sociétés d'agriculteurs sédentaires (Olawale, 1961). En dehors de tout système de garantie des droits au tiers, le lien entre le possesseur et sa possession était établi par l'emprise de l'empreinte vitale du possesseur sur sa possession ${ }^{8}$. Ceci permet de mieux comprendre sans doute pourquoi chez les Dogon comme chez leurs voisins, le vol entre soi était un crime. En effet, dans ce cadre conceptuel, le voleur se rend responsable d'un attentat inique, un crime, contre l'intégrité même du possesseur de la chose ${ }^{9}$. De ce point de vue, on peut considérer que le vol est le pendant matériel de l'agression sorcière qu'il permet et rend possible! Inversement, le vol des "étrangers", toujours potentiellement ennemis, était considéré comme un acte de bravoure qui conférait de l'honneur au voleur.

17 Entre les membres d'une grande maison, il ne pouvait donc y avoir de vol. Quel que fut le "propriétaire » d'un bien individuellement produit, inventé ou acquis, «son » bien peut à tout instant être réclamé par ses aînés ou utilisé par ses cadets, car ce bien est $a$ priori considéré par tous comme un bien commun aux membres de la grande maison qui participent d'une même force vitale ancestrale. Pour eux, il s'agit de disposer collectivement et de manière prioritaire d'une richesse dont l'usage et la gestion sont séparables de l'individu producteur ou acquéreur car elle est considérée comme inséparable du bien commun auquel participe cet individu.

On retiendra ici que l'acte d'appropriation d'une ressource par un sujet n'était jamais transformé automatiquement en droit de propriété ; le groupe constatait simplement le 
fait d'une prise de possession individuelle. La question qui se posait n'était pas celle de sa légitimité, mais celle de son effectivité. Elle devenait effective, stabilisée, à la mort $\mathrm{du}$ possesseur individuel par l'héritage dont le processus "patrimonialisait " la ressource qui devenait une "propriété commune ", res communes, de la progéniture du défunt. Selon les normes coutumières, c'est par cette transmission que l'appropriation s'accomplissait : une maîtrise exclusive était toujours collectivement détenue, garantie et défendue. Mais comment était-elle garantie et défendue?

\section{La régulation interne aux communautés rurales sédentaires}

19 En effet, toute possession n'était respectée par autrui que pour autant qu'elle était effective, c'est-à-dire visible, revendiquée et défendue par son maître par tous les moyens dont il disposait et en premier lieu par la menace de sanctions magicoreligieuses. La jouissance effective d'une richesse, d'un patrimoine ou d'une possession locale n'était a priori garantie par rien d'autre que la menace de sanctions mystiques exercées par les divinités chtoniennes, les ancêtres ou les fétiches qui protègent les villages et leurs ressortissants (Bouju, 1995). Et en ce qui concernait la terre en Afrique de l'Ouest, on rencontre partout le recours systématique à l'ordalie.

Mais ces menaces magico-religieuses.ne constituaient une protection efficace contre toute tentative de dépossession que dans la mesure où la croyance dans leur efficacité était partagée par l'agresseur. ${ }^{10}$ ! Cette sphère de partage des divinités et des croyances était assez précisément délimitée par les frontières généalogiques du groupe et par celles de son terroir. Mais au-delà des frontières claniques, religieuses ou ethniques, les normes de solidarité ne jouaient plus, les croyances n'étaient plus guère partagées et les sanctions mystiques perdaient leur efficacité. ${ }^{11}$. Partout, l'exclusivité des maîtrises foncières n'était plus garantie ni sécurisée au-delà du niveau cantonal (baginê en langue dogon)!

\section{La régulation externe et intercommunautaire}

21 Au-delà du terroir clanique, avec les "étrangers » à la communauté, le principe de respect du bien d'autrui n'était plus garanti par les sanctions mystiques ${ }^{12}$. et la solidarité faisait systématiquement place à la rivalité. Aujourd'hui encore, du point de vue populaire, il ne suffit pas à quelqu'un d'affirmer la maitrise exclusive d'une richesse (une parcelle, un troupeau, un véhicule) ou celle d'un lien exclusif à une personne (fiancé(e), épouse ou mari) pour qu'elle soit reconnue et respectée en conséquence. Pour être respectée par autrui, et donc acquérir son caractère permanent et inaliénable, une maîtrise exclusive doit être visiblement exercée et défendue contre toute tentative d'appropriation par un tiers. Dès lors, une maîtrise exclusive détenue par un non parent n'est collectivement reconnue et temporairement établie que si son titulaire se montre capable de la défendre ${ }^{13}$. (Bouju et al., 2002). C'est à ce niveau segmentaire qu'apparaît de manière éclatante l'incapacité de fait de la Loi à garantir les droits des citoyens. Nos enquêtes de terrain dans différentes régions du Mali et du Burkina Faso ont montré que toute richesse «bien gardée » (terre, bétail, femme, matériel, etc.) était une richesse effectivement défendue par un maître en mesure d'en interdire l'accès à toute personne non autorisée. Dans ce cas, le maître, sa maîtrise et les richesses maîtrisées étaient respectés par d'autres usagers potentiels. Nous 
retrouvons ici la question qui se posait plus haut, à savoir quels sont les moyens de défense dont on dispose pour faire valoir « ses droits » affirmer et garantir une maitrise exclusive que le système juridico-policier de l'État est incapable de protéger?

Quand l'interaction sociale franchit les frontières communautaires, le principe et le répertoire de régulation qui prévalait dans le cas précédent ne peut plus s'appliquer. Dans ces circonstances, c'est principalement la rivalité (fadenya) d'honneur (horonya) et l'éthique de la honte (maloya) qui régulent l'interaction et qui garantissent la maitrise exclusive des uns vis-à-vis des autres. C'est le sens de l'honneur du «maître », d'une personne ou d'un bien qui défend l'exclusivité de la maîtrise. Ces sociétés d'interconnaissance sont aussi des civilisations de l'honneur et de la honte. Sociétés sans écriture, l'image qu'on donne de soi et celle que renvoie le regard des autres expriment l'intériorisation des normes sociales, l'extériorisation des signes statutaires, le droit à la préséance et la morale qui en découle (Peristiany, 1966). Aujourd'hui encore, dans les milieux populaires ouest africains, c'est le respect du code partagé de l'honneur et de la honte qui garantit, mieux que le Droit, l'exécution réciproque des obligations contractuelles de l'échange (Bourdieu, 1979; Vuarin, 1994 ; Lund, 1999; Bouju, et al., 2004).

Ce code social de l'honneur et de la honte fournit en partie les normes et les critères d'évaluation propres aux sociétés bamakoise ou bobolaise. Dans les milieux pauvres, les échanges sociaux demeurent régulés et garantis par des normes largement partagées de civilité, fondées sur un code général de l'honneur et de la honte ${ }^{14}$. où le Droit - et en particulier l'égalité juridique qui fonde l'Etat de Droit - n'a pas sa place (Bouju et al., 2004). Bien que susceptible de variations mineures ${ }^{15}$, le répertoire normatif de la civilité est dans ses grandes lignes, connu et reconnu, dans toutes les sociétés patrimoniales d'Afrique de l'Ouest et d'ailleurs (Ouattara, 1999). Et c'est par rapport aux exigences, c'est-à-dire aux valeurs - plus ou moins précisément définies selon les circonstances - de ce code social d'honneur et de la honte, que s'évalue le capital symbolique de chacun. L'homme respectable connaît les privilèges et les obligations attachées à sa position sociale et si son modèle de référence est la noblesse, il se réfère aux règles de bienséance fondées sur la pudeur et la discrétion dans ses rapports à autrui. L'honneur est ainsi la qualité d'excellence qui assure la fidélité à la parole donnée, le respect des conventions, des coutumes et des contrats et qui de la sorte, garantit les maitrises d'usage et les propriétés de chacun. L'honneur n'est pas un sentiment, c'est un code social de monstration à autrui de ses préséances et de ses maîtrises statutaires dont on exige la reconnaissance ${ }^{16}$ dans une société où la protection ou la garantie d'une maîtrise n'est pas assurée par le système juridicopolicier de l'État, mais par l'aptitude du maître à défendre son honneur. ${ }^{17}$. Mais comment la réputation d'honneur se construit-elle aujourd'hui?

\section{Voir \& être vu ou la fixation de la valeur sociale de chacun au marché de l'estime}

24 À la suite de Pierre Bourdieu, on analysera l'honneur en termes de capital symbolique. En effet, l'honneur d'un individu dépend de la reconnaissance sociale de son excellence dans cette qualité hautement valorisée culturellement qu'est la capacité à garantir l'invulnérabilité des "points d'honneurs " que représentent les patrimoines et les richesses maîtrisées (domaine, terre, hommes, femmes, bétail, argent, etc.) susceptibles d'être transmis ou échangés (Bourdieu, 1979 : 205). 
La reconnaissance sociale de l'excellence qui honore n'est pas facile à gagner. Elle est le résultat d'un travail permanent de mise en scène de la valeur de soi aux yeux des autres, travail qui permet d'accumuler le capital symbolique sous la forme du renom, du prestige, de la réputation. L'espace sociopolitique local construit par cette logique aboutit à une sorte de "marché de l'estime » (Vuarin, 1994) dans lequel les jeux sur les différents sens de la considération peuvent se développer. La considération de soi vers soi d'abord, celle des autres vers soi ensuite et puis, surtout, celle de soi vers les autres, guettant le regard des autres sur soi. Ainsi, finit-on par n'exister socialement que par le regard qui est porté sur soi : il faut voir et il importe au plus haut point d'être vu. Dans un tel contexte, la qualité d'homme d'honneur est d'abord une affaire de regard et de visibilité $^{18}$. des actes dans la sphère d'interconnaissance.

\section{Le défi ou le point d'honneur attaqué}

26 À cet égard, la reconnaissance collective n'est pas accordée à celui qui est économe ou respectueux des maîtrises d'autrui, mais à celui qui, au contraire, peut étaler sa maitrise sans limites d'un bien prestigieux. Le corollaire est que l'autorité d'un pouvoir - posséder la maîtrise exclusive d'une ressource est une forme de pouvoir - n'est reconnue que dans la mesure où ce pouvoir se montre capable de maintenir son exclusivité en contrôlant effectivement l'accès aux richesses qui constituent sonhonneur.

En tant que capital symbolique, c'est-à-dire crédit d'excellence accordé par la société, l'honneur peut être conféré ou acquis, attaqué, défendu ou perdu ${ }^{19}$. Car, l'honneur s'acquiert aussi en défiant l'honneur des autres ${ }^{20}$, par exemple en accaparant la propriété d'autrui : «La personne qui ne mettrait jamais en danger la propriété ou l'honneur de son semblable ne peut pas être considérée comme ayant de l'honneur par elle-même" (Peristiany, 1966). On admire celui qui arrive à s'emparer du "bien d'autrui » et à en profiter sans se faire prendre, car il fait ainsi preuve d'une grande capacité de maitrise et l'on méprise celui qui se fait déposséder de son bien car cela veut dire qu'il est incapable de le maîtriser : « il ne vaut rien, il n'est même pas capable de défendre sa famille et son bien!» (Lund, 1999).

La honte est l'état dans lequel se trouve la personne discréditée par ses pairs et donc déshonorée parce qu'elle a manqué à l'obligation d'honneur qui consiste à se défendre contre les attentats à ses richesses. Mais la honte est moins la conséquence de la conscience individuelle d'avoir manqué à ses devoirs, que la découverte par le regard des autres ou la révélation publique de ce manquement. Comme la discrétion et la dissimulation aux regards des autres sont la meilleure protection contre les diverses sortes d'attentats aux points d'honneur, il se développe alors une "rhétorique de la dénonciation » et une " casuistique du soupçon » pour identifier sous les artifices de la double pensée ${ }^{21}$. l'État de d'honorabilité dans lequel les autres se trouvent.

\footnotetext{
"Au principe des conduites d'honneur se trouve "l'intérêt symbolique »: toute famille a un intérêt vital à tenir son capital d'honneur (son crédit d'honorabilité) à l'abri de la suspicion. La susceptibilité, la sensibilité aux moindres allusions s'explique par le fait que le capital symbolique ne se laisse pas aussi facilement mesurer que la terre ou dénombrer que le bétail. Ensuite, le groupe de référence qui peut seul l'accorder - est toujours prêt à retirer sa confiance (sa croyance) en portant ses soupçons sur les plus grands comme si, en matière d'honneur,
} 
l'enrichissement de l'un ne pouvait se faire qu'au détriment des autres.» (Bourdieu, 1979 : 205).

- devrait provoquer de sa part une réaction de vengeance qui vise à réparer l'offense faite à sa réputation d'honneur. Quand bien même l'offense ne serait pas grave, la réaction doit être dissuasive, car toute violation de l'honneur est une atteinte potentielle à la totalité des biens possédés par la partie lésée.

«Le patrimoine lignager, symbolisé par le nom, n'est pas défini seulement par la possession de la terre, de la maison et des filles, biens précieux et donc vulnérables, mais aussi par les moyens de les protéger: les hommes; des attaques contre la terre, la maison et les femmes sont des attaques contre leur maître. Terres accaparées, meurtres et viols non vengés sont des variétés de la même offense. L'honneur est lavé par la vengeance, par un meurtre plus signifiant: une personne plus proche du meurtrier ou la plus représentative du groupe meurtrier, terre reconquise à n'importe quel prix, effacent l'insulte faire à l'honneur du groupe » (Bourdieu, 1966)

\section{L'honneur contre le Droit !}

30 Le code général de l'honneur et de la honte joue toujours un rôle considérable dans la régulation des échanges sociaux, ce qui pose l'importante question de la légitimité du Droit. En effet, dans un espace social régi par le code de l'honneur, celui-ci est éminent, et toute personne jugée honorable par ses pairs peut «sans aucune perte d'intégrité, transgresser d'autres règles sociales qui, par comparaison, sont toujours considérées comme moins importantes. » (Péristiany, $1966: 10)$.

31 La question de la soumission consentie à la Loi et au Droit en général est donc posée. Ainsi, dans le contexte de relative impunité légale qui règne à Bobo-Dioulasso ou à Bamako, le code de l'honneur établit une hiérarchie des «bonnes » conduites, et de leurs sanctions effectives, qui ouvre la possibilité d'agir contre les normes légales. Comme le citadin craint plus la vengeance de ses pairs que la sanction du tribunal, Il devient possible de transgresser la Loi et les règlements communaux tout en conservant son honneur et sa bonne réputation. Ce point est capital, car il permet, je crois, de mieux comprendre le rapport social que les citadins entretiennent avec les lois, les normes et les règles de toutes sortes, qui sont à la base du pluralisme normatif et de l'anomie urbaine.

\section{La situation actuelle : la généralisation des rapports prédateurs}

L'acuité des conflits fonciers régionaux traduit bien la pression foncière formidable qui s'exerce aujourd'hui tant dans l'espace urbain des capitales que dans les terroirs villageois. L'instabilité générale et la confusion des normes en matière de «droits fonciers traditionnels " soulignent dramatiquement l'incapacité des répertoires normatifs traditionnels à y faire face. Toute ressource est l'objet de tentatives d'accès et d'usage. On ne sait jamais! Peut-être que les maîtres sont corruptibles ou peut-être sont-ils démunis au point d'avoir perdu leur capacité de défense mystique. L'absence de réaction immédiate du «maître » d'une ressource foncière est toujours perçue comme une faiblesse et donc comme un encouragement à aller de l'avant et à pousser son 
avantage jusqu'à ce que l'espace soit privatisé, jusqu'à ce que l'espace limitrophe de la cour soit complètement occupé ou jusqu'à ce que l'accès à la mare soit envahi par les cultures. Avec le développement de la prolifération normative, il semble que cette instabilité se soit dramatiquement aggravée. En témoignent deux phénomènes urbains que nous avons pu étudier: d'une part, la privatisation des passages publics par les riverains et d'autre part, la spéculation foncière dans les quartiers périphériques des grandes villes.

\section{La privatisation des lieux publics urbains par les usagers}

Aujourd'hui, l'explosion démographique des grandes villes conjuguée aux défaillances des autorités urbaines chargées de la régulation de contrôle (mairie, voirie, police, gendarmerie, système judiciaire) est telle que les citadins n'ont plus en partage la dimension impérative des normes de vie en commun. Mise à part la « loi » du plus fort, aucune norme partagée ne joue plus ce rôle de repère idéal en référence auquel chacun se sent en droit d'évaluer les actions conformes et donc de porter des jugements de valeur sur les actes d'autrui ${ }^{22}$. La «loi» du plus fort tend à s'imposer dans tous les domaines d'interaction. Ainsi, vis-à-vis des espaces urbains considérés, à tort ou à raison, comme laissés en libre accès, la privatisation informelle tend à se généraliser. En ville, la plupart des citadins des milieux populaires considèrent l'espace public urbain immédiatement au-delà du mur d'enceinte de leur concession d'habitation comme un domaine en libre accès où peut s'exercer et s'affirmer leur liberté individuelle d'agir.

«Les espaces limitrophes vacants sont annexés à la cour dans un processus d'appropriation qui est en général lent, prudent et progressif quant aux marques produites. On guette surtout la réaction des autorités ou des voisins et l'absence de réaction est toujours interprétée comme un signe d'acceptation tacite qui encourage éventuellement le renforcement progressif des marqueurs matériels.

L'observation des pratiques sociales permet de distinguer deux modalités principales d'occupation de l'espace limitrophe. La première correspond à une extension du logement qui se manifeste par une appropriation de l'espace pour des usages qui appartiennent à la sphère privée de la cour et qui sont, soit occasionnellement, soit durablement déployés dans l'espace limitrophe. Ainsi, le jour, l'espace est occupé par des activités féminines : lavage (lessive, vaisselle, tri et lavage des céréales) tablier et coupe du bois. Ensuite, mais à d'autres moments du jour, il est le lieu des activités des hommes, orientées plutôt vers le repos ou la sociabilité : prendre le thé avec son grin, jouer à la pétanque, la belote, les dames ou l'awalé. Les enfants y jouent et les plus grands bavardent entre voisins. La sociabilité y est soumise au jeu dialectique de l'ostentation et de la discrétion et son appropriation sociale est ambivalente. Cette occupation, cette appropriation de l'espace limitrophe et du "six-mètres. ${ }^{23}$. peut être vue comme une tentative chaque jour renouvelée de réinventer un domaine commun à plusieurs familles qui, entre le domaine privé et le domaine public, faisait toute la richesse de la sociabilité africaine de proximité. La seconde modalité se manifeste par un marquage du territoire qui consiste à prendre des dispositions matérielles ou non pour constituer l'espace limitrophe en un "dehors de la limite habitée " tout en le soustrayant à l'usage que pourraient en faire les autres. Ainsi, en dehors des périodes d'occupation effectives, les usages de l'espace limitrophe donnent lieu à quelques marquages symboliques et temporaires du territoire. Il en va ainsi, des tables des « restaurants par terre » que l'on retourne à l'envers pour la nuit, de l'entrepôt de matériel de construction ou de la construction d'un hangar pour abriter un petit 
commerce. Évidemment, cette invention se fait au détriment de l'espace public, mais comme la puissance publique est absente et désespérément silencieuse... En situation de bon voisinage, un consensus règne autour du respect de l'espace limitrophe de la cour voisine. L'usage de l'espace limitrophe est un bon indicateur de la qualité des relations de voisinage. Quand les relations sont bonnes, l'usage privatif ne se fait pas de manière anarchique, il se fait dans la connivence avec les voisins et dans le respect du code de civilité partagé. » (Boujuet al., 2004 : 24-25)

L'appropriation de l'espace limitrophe résulte donc, tout à la fois, d'un «coup de force " (on ne demande la permission à personne) et d'un accord tacite entre voisins sur sa possibilité (car personne ne se plaint). Ces actes de privatisation de l'espace limitrophe n'empêchent pas qu'il soit reconnu par les gens comme un espace public et comme tel, les gens attendent de la puissance publique qu'elle se charge de son aménagement et de son entretien. À cet égard, les manques et les insuffisances des services municipaux qui se traduisent par l'absence d'éclairage public, de goudron sur la voie, de trottoirs, d'adduction d'eau potable, d'entretien des caniveaux, se ressentent encore plus dans l'espace limitrophe qui est vécu au quotidien. Quoi qu'il en soit, les pratiques et les usages quotidiens des habitants recomposent ainsi la signification des lieux en déconstruisant la structure imposée de l'habitat urbain par rapport auquel les activités se redistribuent.

Pour la plupart des Bobolais, mais il en va de même à Bamako, tout espace public est considéré un espace en libre accès. À Bobo-Dioulasso, on peut régulièrement en observer les effets à travers le traçage des chemins piétonniers en particulier dans les quartiers périphériques. La voie de circulation pratiquée par les gens dans la rue est souvent moins large que le six-mètres, elle forme alors un sentier emprunté par les cyclistes et les véhicules qui zigzaguent entre les trous d'eau, les dépotoirs d'ordure et les arbres. Mais elle est beaucoup plus large parfois quand elle emprunte les terrains vagues. Les déplacements se font en fonction des sentiers pédestres et cyclistes tracés par l'usage en fonction des traces laissées par l'écoulement des eaux pendant l'hivernage et en fonction des parcelles et des espaces non mis en valeur. Les chemins pratiqués par les piétons et les motos sont si bien ancrés dans les pratiques des habitants qu'ils acceptent mal de devoir dévier leur route quand le mur d'enclos d'une construction apparait sur un lotissement ${ }^{24}$. Tant et si bien que si l'attributaire de la parcelle « n'a pas les moyens de construire très vite, les pierres sont poussées, les fils de fer écartés, puis retirées, et le droit de passage revient aux usagers... » (Déverain-Kouanda, 1991 : 95). Pour les citadins, un espace de circulation (une rue, un trottoir, une place) est simplement un espace vide, comme s'il n'y avait aucune autorité qui s'exerce dessus ! (Bouju et al. 2004:25)

Le citadin s'approprie donc des portions d'espace public urbain à travers un usage individuel qui a pour corollaire l'exclusion d'autres usagers potentiels tant que dure l'utilisation de cette portion d'espace. Si quelqu'un tente d'objecter à ces tentatives de privatisation de l'espace public, la réponse de «l'envahisseur » est toujours la même : «ce n'est pas pour ton père!». Cette petite phrase, maintes fois entendue, confirme notre analyse. Elle pose comme principe que le pouvoir de dire quelque chose sur l'usage d'une ressource est lié à l'exercice effectif d'une maîtrise exclusive sur cette même ressource. Or, selon l'habitus populaire, la première caractéristique des lieux publics de la ville est de n'appartenir à aucune famille, donc à personne. Ce qui ne signifie pas qu'ils appartiennent à tout le monde, car ce serait alors un «bien public ». Or, la conception d'un bien public qui serait commun à toutes les familles de la ville n'existe pas ${ }^{25}$ ! Bien au contraire, si l'espace public n'appartient à personne, il est donc, selon la coutume, en libre accès; c'est-à-dire utilisable privativement par toute personne capable de s'en saisir pour en faire un usage privé ! Autrement dit tout espace 
non utilisé ${ }^{26}$. est considéré comme potentiellement utilisable par n'importe quel usager, quel qu'en soit par ailleurs le propriétaire! On retrouve ici la même conception de la maîtrise foncière selon laquelle la légitimité d'une maîtrise d'usage d'une ressource est directement fonction du pouvoir mis en œuvre par son maître pour la défendre. La réussite visible des pratiques populaires témoigne de l'impuissance des autorités communales à défendre leur conception de l'ordre urbain (Bouju, 2002).

\section{La spéculation foncière dans les périphéries urbaines}

36 La généralité de la spéculation foncière dans les périphéries en voie de lotissement et la gravité des détournements de parcelles d'habitation que nous avons pu observer dans les communes de Bamako (Mali) et de Bobo-Dioulasso (Burkina faso) témoignent du même phénomène. En effet, à travers la parcellisation du territoire communal par les lotissements urbains, c'est tout un système de privatisation illégale de l'espace public urbain qui s'est ainsi mis en place avec la décentralisation (Bouju et al., 2004).

La généralité et l'extension du phénomène montrent à quel point les parcelles d'habitation urbaines sont considérées comme une richesse dont la possession est ardemment désirée et recherchée par tous les citadins. Parce qu'elle est un refuge et parce qu'elle soustrait les hommes aux agressions du monde extérieur, la concession d'habitation qui abrite la maison n'est jamais une richesse économique quelconque, ni une simple marchandise. Aujourd'hui, la détention d'un capital foncier et immobilier est, plus que jamais, devenue un élément central des stratégies de reproduction ou d'accumulation des chefs de famille. Tout chef de famille qui en a les moyens, développe une stratégie d'acquisition de parcelles à construire sur l'ensemble du territoire urbain de la ville, et souvent même, dans les autres villes du pays !

Cette stratégie des chefs de famille a pour objectif de prendre des gages fonciers un peu partout, de manière à perpétuer leur autorité et à renforcer leur capacité redistributive au sein de leur propre famille, mais aussi à se prémunir contre un déguerpissement administratif toujours possible. C'est l'importance de cette demande sociale qui explique pourquoi les parcelles sont devenues en enjeu si important du clientélisme politique communal ces dix dernières années. La possession légale ${ }^{27}$. garantit non seulement la jouissance d'un refuge, mais aussi, et surtout, la possibilité pour le « chef " de cour de retrouver une sécurité statutaire en se positionnant comme patron dans un rapport de domination clientéliste durable (à travers, par exemple, l'hébergement des juniors cadets : frères, fils, neveux), ou tout simplement une sécurité financière en captant une rente locative en louant la cour à des « étrangers ». Inversement, pour un cadet, la possession d'une cour permet de se soustraire au risque d'être dominé indéfiniment par un grand frère hébergeur ou un père propriétaire.

Une parcelle peut ainsi être affectée à la résidence d'un fils marié avec lequel on ne veut pas rompre mais dont on sent bien qu'il éprouve le besoin de prendre ses distances vis-à-vis d'une cohabitation communautaire vécue comme trop pesante. En quittant de manière non conflictuelle la cour paternelle à la tête de leur famille nucléaire, les juniors cherchent à s'émanciper et gagner leur autonomie; mais en acceptant malgré tout la parcelle offerte par le père, les cadets prennent des distances spatiales vis-à-vis de la famille ${ }^{28}$. tout en demeurant les obligés du père. On assiste ainsi à des prises de 
distance très subtiles qui permettent aux juniors-cadets de sauver la face de leurs seniors-aînés.

Les terrains prévus pour les lotissements dans la périphérie des grandes villes sont presque toujours des patrimoines fonciers «traditionnels » et, à Bobo-Dioulasso, ils appartiennent aux clans des maîtres de la terre Bobo. Cependant, les aînés représentants ces clans qui détiennent la maîtrise exclusive du patrimoine foncier n'ont pas hésité à vendre clandestinement à des chefs de familles citadines en quête de logement, et à titre privé, des maitrises d'usage de parcelles situées sur le domaine foncier commun dont ils ont "traditionnellement» la garde, sans en informer, bien sûr, les ayants droit légitimes. Il s'agit là d'un acte de corruption caractérisée qui devait inaugurer une longue série d'actes semblables. Mais les problèmes surgirent quand la municipalité se porta acquéreur du domaine. En effet, pour être en mesure de lotir tranquillement la zone, l'équipe municipale récemment élue a racheté aux maitres de terre de Bobo (pour une somme dérisoire) ce même patrimoine foncier qui a ainsi été revendu une seconde fois par les mêmes autorités coutumières !

41 Le scandale éclata en plein jour quand les équipes du cadastre municipal voulurent procéder au bornage des futurs lotissements; les villageois Bobo s'en sont rendus compte et ont découvert le pot-aux-roses. C'est ainsi que dans les terroirs villageois de Sakaby et de Kwa qui sont atteints par les limites de la ville de Bobo-Dioulasso, le conflit a fait rage et les bornes parcellaires installées par le service du cadastre ont été arrachées par les paysans furieux d'avoir été floués par leurs propres frères. Malgré tout, le processus de parcellisation de la zone s'est poursuivi inexorablement et les attributions foncières ont commencé.

42 Le nombre de parcelles loties n'étant pas connu du public, le choix final par la mairie des attributaires de la «manne » parcellaire s'est fait sur la base d'une redistribution dans la clientèle des principaux notables locaux et décideurs politiques municipaux, associés depuis le début au processus de lotissement: le Maire, les principaux Conseillers Municipaux, le Gouverneur Régional, etc. Comme dit le proverbe bobolais : «Tous les oiseaux mangent du mil, mais celui qui s'installe dans le champ de mil pour manger, on l'appelle mange-mil ».

Le cycle de l'échange clientéliste municipal pouvait alors s'accomplir. En tant que patron, chacun des attributaires de parcelle pouvait en redistribuer à ses clients, « ceux qu'il aide» et, en tant que client, chacun pouvait offrir une parcelle à ses patrons « ceux qui, parmi les notables nationaux ou locaux, l'ont aidé». Les premiers servis dans cette "grande bouffe» foncière furent les autorités ministérielles qui avaient délivré l'autorisation de lotir. Les seconds furent les membres de la commission de lotissement eux-mêmes et enfin, les services communaux du cadastre, des travaux publics, etc. Au Burkina Faso, au Mali, les élus municipaux et les principaux responsables des services déconcentrés concernés par les lotissements se sont discrédités par leur implication dans ces processus d'appropriation illégale de parcelles d'habitation. À vrai dire, ce capital foncier urbain est devenu leur principale source de ressources. C'est lui qui alimente et entretient la sphère d'échange du clientélisme politique local, et comme nous confiait un ancien Conseiller Municipal « La gestion des lotissements est le « bon » qu'il y a " à manger » dans une élection municipale ».

Ce phénomène de marchandisation brutale de patrimoines fonciers et de domaines ancestraux est tellement répandu au Burkina Faso et au Mali, et sans doute ailleurs, qu'il mérite qu'on s'y arrête quelque peu. En l'occurrence, il s'agit bien d'une forme de 
corruption puisqu'il y a une transaction sociale clandestine, illégitime en termes coutumiers, par laquelle les représentants des clans maîtres de la terre "négocient " leur capacité de gestion exclusive (exercée en vertu de leur autorité coutumière) contre des bénéfices économiques personnels. C'est donc indéniablement une transaction corrompue.

Mais cette aliénation brutale du bien commun est, plus que d'autres, extrêmement lourde de conséquences. En effet, c'est par le truchement de la corruption des chefs coutumiers que le patrimoine foncier commun à un collectif clanique a été " métamorphosé » en marchandise par son découpage en parcelles géométriquement bornées et appréciées. ${ }^{29}$. La corruption des chefs coutumiers Bobo est une sorte de trahison de leur communauté. Par cet acte d'aliénation, ce n'est pas seulement la maitrise commune d'un patrimoine foncier qui est définitivement perdue, c'est l'identité même des agriculteurs Bobo concernés, ceux des clans de Kwa et de Sakaby qui sont devenus dans ce processus, et malgré eux, de simples cultivateurs: des paysans.

le processus délétère ne s'arrête pas là. Selon la loi, l'acquisition par la municipalité de ce bien commun aurait dû le transformer en bien public communal. Or la corruption qui nourrit la logique clientéliste du pouvoir municipal est telle qu'il sera directement converti et métamorphosé en bien privé, c'est-à-dire en marchandise clandestinement et illégalement appropriée et accumulée par les divers détenteurs de l'autorité publique communale. Cette forme de corruption suppose comme condition nécessaire à sa généralisation l'existence d'une conception néopatrimoniale ${ }^{30}$. et comme condition suffisante que cette confusion ne soit pas considérée comme illégale, ou illégitime, par les acteurs sociaux.

Mais qui va protester officiellement contre cette confusion? Tout le monde bien sûr se plaint de la corruption; pourtant chacun est, selon les circonstances du moment, patron ou client, corrupteur ou corrompu. « La grande bouffe » politico-économique se redistribue le long des réseaux d'appartenance communautaires, elle circule par le canal des relations de parenté et d'alliance ou de solidarité villageoise, tribale, ethnique et régionale. C'est aussi par ces relations que transitent les transferts de revenus des citadins aux parents du village, des plus riches aux plus démunis. Quel répertoire normatif éthique ou juridique pourrait être considéré aujourd'hui comme légitime et convoqué par la majorité des citadins?

Dans ce jeu inégal, mis à part les élus municipaux et leurs clients, tout le monde a perdu. Les collectifs communautaires ont perdu leur «trésor », le patrimoine foncier qui fondait et matérialisait leur identité collective et les citoyens de la ville ont perdu le bien public qui aurait pu fonder et matérialiser leur identité citoyenne. Car ces pratiques prébendières illicites sont en contradiction avec le service de l'intérêt général associé à l'exercice des fonctions politiques et bureaucratiques. La métamorphose des maitrises exclusives collectivement détenues sur des domaines patrimoniaux en propriétés foncières marchandes individuelles est un événement majeur dans l'histoire récente de l'Afrique. 


\section{BIBLIOGRAPHIE}

Bouju, Jacky, 1984, Graine de l'homme. Enfant du mil, Société d'ethnographie, Coll. Sociétés Africaines, vol. 6.

Bouju, Jacky, 1995, « Fondation et territorialité. Instauration et contrôle rituel des frontières (Dogon Karambé, Mali) », in Daniel DORY et alii (éds), La construction religieuse du territoire, Coll. Connaissance des hommes, L'Harmattan, Paris : 352-365.

Bouju, Jacky, 1998 « Contrôle foncier \& conflits pour les ressources : l'accès aux bas-fonds aménagés comme enjeu de pouvoir local », Aménagement et mise en valeur des bas-fonds au Mali, Actes du Séminaire de Sikasso,Montpellier : Editions du CIRAD/CBF : 95-108.

Bouju, Jacky, 2004, « Le coutumier juridique africain : approche anthropologique d'une invention coloniale » in Dejan DIMITRIJEVIC (éd.), Fabrication de traditions, invention de modernités, Paris, Editions de la Maison des Sciences de l'Homme : 125-152.

Bouju, Jacky et Ouattara Fatoumata (dir.), 2002, Une anthropologie politique de la fange. La souillure de la ville par les eaux usées et les excrétas à Ouagadougou et Bobo-Dioulasso (Burkina faso). Programme de recherche " gestion durable des déchets et de l'assainissement urbain », Action de recherche $\mathrm{n}^{\circ} 4$,SHADYC (Marseille)-GRIL (Ouagadougou) : 224 pages. Annexes : 86 pages, photos, cartes.

Bouju Jacky (dir.), Ouattara Fatoumata, Bocoum Hambarke, Toure Laurence, 2004, Les incivilités de la société civile. Espace public urbain, société civile et gouvernance communale à Bobo-Dioulasso et Bamako (Communes 1 et 2), Programme de Recherche Urbaine pour le Développement, Paris, GEMDEVISTED : $169 \mathrm{p}$.

Bourdieu, Pierre, 1966, « The sentiment of honor in Kabyle society » in J. G. Peristiany (dir.) Honor and Shame : the values of mediterranean society, Chicago, University of Chicago Press : 191-241.

Bourdieu, Pierre, 1979, La distinction, Paris, Editions de Minuit : 244 p.

Cohen-Tanuggi, Laurent, 1985, Le droit sans l'Etat, PUF/recherches politiques.

Handman, Marie-Elisabeth, 1991, « Propriété » in Pierre Bonte et Michel Izard (Eds) Dictionnaire de l'Ethnologie et de l'Anthropologie, Paris, P.U.F. : 605-606

Lamaison, Pierre, 1991, « 2 - Les formes de la transmission » in Dictionnaire de l'Ethnologie et de l'Anthropologie, Pierre Bonte et Michel Izard (Eds), Paris : P.U.F. : 713-714.

Le Bris, Emile, Le Roy, Etienne, Leimdorfer, François(Dir.), 1983, Enjeux fonciers en Afrique noire, Paris, Karthala, 1983 : 425 p.

Le Roy, Etienne, 1996, « La théorie des maîtrises foncières » in Le Roy, Karsenty et Bertrand (Dir.), La sécurisation de la terre en Afrique Noire. Pour une gestion viable des ressources renouvelables, Paris, Karthala : 59-76.

Le Roy, Etienne, 1998, «L'hypothèse du multijuridisme dans un contexte de sortie de modernité ", Théorie et émergence du droit, pluralisme, surdétermination et effectivité, in Lajoie,

Macdonald, Janda et Rocher (Dir.), Montréal, Editions Thémis, Bruxelles Editions Bruylant : 29-44.

Lund, Christian, 1999, "A Question of Honor : Property Disputes and Brokerage in Burkina faso », Africa, 69, (4) : 575-594.

Madjarian, G., 1991, L'invention de la propriété. De la terre sacrée à la société marchande, Paris, L'Harmattan : $271 \mathrm{p}$. 
Olawale, Elias T., 1961 La nature du droit coutumier africain, Présence Africaine, « Enquêtes et études ». Manchester : Manchester University Press.

Ouattara, Fatoumata, 1999, Savoir-vivre et honte chez les Senufo Nanerge (Burkina Faso), doctorat nouveau régime, Marseille, Ecole des Hautes études en Sciences Sociales.

Sandberg, A., 1994, « Gestion des ressources naturelles et droits de propriété dans le grands nord norvégien : éléments pour une analyse comparative », Nature - Science - Sociétés, 2 (4) : 323-333

Schlager, Edella \& Ostrom, Elinor, 1992, « Property Rights Regimes and Natural Resources : a conceptual analysis », Land Economics, 68 (3), august : 249-62.

Verdier, Raymond, 1986, "Civilisation paysanne et traditions juridiques », in Verdier Raymond et Rochegude Alain (Eds), Systèmes fonciers à la ville et à la campagne, l'Harmattan.

Vuarin, Robert, 1994, L'argent et l'entregent à Bamako. Cahiers des Sciences Humaines, 30 (1-2) : 255-273.

\section{NOTES}

1. En anthropologie juridique, la "possession » est une "propriété de fait », c'est-à-dire sans avoir de titre reconnu de propriétaire qui puisse faire preuve au tiers. La «propriété » renvoie à la «possession de droit »c'est-à-dire la revendication de bénéfices à venir sur une ressource donnée.

2. Il est clair que dans nombre de régions de l'Afrique rurale cette conjonction était loin d'être achevée au moment de la colonisation.

3. L'anthropologie juridique n'a guère distingué qu'entre propriété de biens matériels et immatériels (Lowie R.H., 1928 "Incorporeal Property in Primitive Society » Yale Law Journal, XXXVII, 5 : 551-563) ou propriété collective et individuelle (Mauss, 1985 ; Godelier, 1984).

4. - Une cinquième maîtrise, prééminente, concerne le droit d'abusus ou d'aliénation qui apparaît quand la terre devient un bien c'est-à-dire une marchandise ayant un prix.

5. On trouve d'excellentes descriptions ethnographiques des rites propitiatoires dans les monographies classiques (purification de la terre, bénédiction des semences, désacralisation de la récolte, etc.).

6. « « Un patrimoine est, par définition, inaliénable (ce qui le distingue fondamentalement de la propriété) et il est de nature intergénérationnelle (on le doit inaltéré aux générations suivantes) (...) il a un caractère permanent, et il est intimement lié à l'identité de ses titulaires dont il est une composante essentielle. » (Le Roy et al., 1996 : 311).

7. - Il est amusant de noter que l'économie classique recourt elle aussi à la puissance métaphorique de la main pour désigner l'emprise sur un bien.

8. Cette empreinte « animée » du possesseur sur l'objet possédé est cohérente avec le principe magique de continuité (ou de contagion) mis en évidence par Hubert et Mauss (Essai sur la nature et la fonction du sacrifice, 1899). Olawale (1961) rapporte que dans la vente d'une vache, une lance était donnée en paiement de «la queue de l'animal»; dans la transaction, la force vitale du vendeur devait être retirée de la vache en passant par la queue pour se concentrer dans la lance offerte. De même, les objets inséparables de l'identité sociale et du statut d'un défunt important devaient disparaître dans le même tombeau que lui. Chez les Dogon, le concept de nyama traduit cette notion « d'empreinte » vitale.

9. Les informateurs expliquent qu'à partir de ce moment on peut tenir la personne sous son emprise et agir sur elle. 
10. Le champ de la morale est confiné à la sphère communautaire et de manière fort contrastée, lesrelations intercommunautaires sont par contre régies par des principes d'amoralité (utilitarisme, prédation, etc.) (Ranger, 1993).

11. Traditionnellement, ces relations extracommunautaires étaient régulées de manière pragmatique et opportuniste; elles relèvent pour partie, de la rivalité d'honneur et pour partie, de l'issue donnée aux litiges et aux conflits passés. N'oublions pas que la coutume est le fait et le privilège d'un groupe limité d'individus: elle ne vise et ne protège que les membres de la communauté.

12. Du fait de l'altérité clanique, les divinités ne sont plus partagées et l'ordalie devient inefficace. Cependant, la réinterprétation de ces procédures de protection par les adeptes des religions monothéistes sous la forme des figures contemporaines de la « sorcellerie » que sont le « maraboutage » ou la « magie noire » étend sensiblement l'espace social d'efficacité des menaces mystiques.

13. C'est à ce niveau que se révèle de manière éclatante l'incapacité de fait du Droit positif de l'État à garantir les droits des citoyens. Nos recherches de terrain dans différentes régions du Mali et du Burkina Faso ont montré que toute richesse «bien gardée » (terre, bétail, femme, matériel) était une richesse effectivement défendue par un maître qui était en mesure d'en interdire l'accès à toute personne non autorisée. Alors seulement, le maître, sa maîtrise et ses biens étaient respectés par d'autres usagers potentiels.

14. - "L'honneur et la honte sont la préoccupation constante des individus dans les sociétés exclusives de petite taille, où les relations personnelles, de face à face, opposées à anonymes, sont d'une importance cruciale et où la personnalité sociale de l'acteur est aussi signifiante que sa fonction. » Peristiany (1966:11). Les sociétés éloignées du contrôle et de la protection de l'État ont développé un « code social » qui peut assurer simultanément l'intégrité de l'individu et de la famille vis-à-vis du reste de la communauté et une certaine solidarité dans la communauté contre le reste du monde (Lund, 1999 : 576).

15. D'une société à l'autre, celui-ci varie en fonction du rang et du statut de la personne. Ainsi, selon le rang de noblesse, d'aînesse ou de séniorité, selon le statut et aujourd'hui, la richesse, le répertoire normatif distingue traditionnellement des qualités honorables différentes qui sont attendues dans les conduites des personnes occupant (ou revendiquant) ces positions sociales. Ainsi, le «noble-guerrier » doit montrer son courage, son arrogance et se faire craindre: «la première qualité est le courage : 'si vous laissez l'adversaire vous insulter en ne répondant pas quand il vole votre terre ou votre bétail, personne ne vous craindra ni ne vous respectera et la prochaine fois, on vous prendra tout.' (...) Il arrive que des gens cherchent des adversaires afin de prouver leur courage.» (Lund, 1999: 585). En la matière, les stéréotypes les plus largement répandus posent que le «noble-régnant » doit faire preuve de générosité et de discrétion; que le «religieux» doit montrer sa piété et sa sagesse; que le "puissant-patron-riche " fasse ostentation de sa générosité, de sa force et de son entregent ; tandis que le « subordonné-clientpauvre » doit faire preuve de respect, de soumission et montrer sa honte, etc.

16. À Bamako ou à Bobo-Dioulasso, honorer un homme, c'est connaître ou reconnaître que cet homme détient un surplus de valeur par rapport à celui qui se mesure ou se compare à lui.

17. - Les citoyens n'attendent pas de l'État qu'il les protège, mais comptent sur leur honneur pour contrer leurs adversaires (Lund, 1999 : 594). L'incapacité de l'État à faire respecter une loi ou un règlement, laisse ainsi le champ libre à la régulation des conduites sociales par le code de l'honneur et de la honte.

18. Ainsi, la transgression des interdits et le manquement à certaines obligations sociales ne seront pas connus - mêmes si ils sont sus - tant qu'ils ne seront pas visibles. Leur visibilité serait infamante et appellerait une sanction collective d'évitement ou de sarcasme qui donnerait 
la honte, discréditerait, atteignant ainsi la réputation d'honneur qui isole et finalement affaiblit la personne par déperdition d'énergie. Ces sociétés ont développé au plus haut point la dichotomie entre l'espace privé (des actes individuels) et l'espace public (où les actes sont donnés à voir).

19. Christian Lund (1999) à la suite de Stewart (1994), de Péristiany (1966) et de Bourdieu (1979), interprète à juste titre l'honneur comme un droit. Le code de l'honneur est une manière de réclamer ou de rechercher la reconnaissance d'autrui, de montrer sa reconnaissance à autrui ou au contraire de ne pas reconnaître autrui en le méprisant (Lund, 1999 : 575).

20. Il ne suffit pas d'être honorable par simple naissance : la réputation d'honneur ne peut pas se maintenir par un conformisme passif aux normes sociales.

21. Ce qu'on croit en son for intérieur et ce qu'il convient qu'on croit en fonction de son rang.

22. Tout espace social commun ou public partagé est " normalement » structuré par des normes dont la fonction est d'imposer une manière d'agir commune et d'assigner une direction commune à l'existence en société. À défaut, on se trouve dans une situation anomique.

23. Six mètres est la distance réglementaire des voies de circulation séparant des concessions ou des parcelles d'habitation. Les autorités communales concèdent à chaque propriétaire de parcelle l'usage d'un mètre devant le mur de sa cour, qui constitue sa « devanture ».

24. - Le dernier exemple en date de ce genre de conduite est fourni par le gigantesque et magnifique mur d'enceinte qui enclot le périmètre du musée national tout en haut de l'avenue Charles de Gaulle à Ouagadougou. Comme le musée tardait à sortir de terre, les usagers des chemins qui traversaient cet immense périmètre avant la construction du mur avaient décidé de reprendre leur route et pour ce faire, ils ont tout simplement rouvert leur sentier en pratiquant deux ouvertures dans le mur qui ont été rebouchées quelques temps après par les pouvoirs publics.

25. Par contre la notion de bien commun, à une grande famille ou à plusieurs familles, existe.

26. Selon les normes populaires d'occupation de l'espace. Ainsi, au village, mis à part quelques lieux sacrés frappés d'interdits, l'espace extérieur à la concession a toujours, et partout, été appropriable en fonction de l'utilité: tout espace non utilisé par quelqu'un est donc éventuellement " propre » à accueillir les déchets

27. L'impression dominante qui ressort de l'ensemble des discours est que les citadins ont une idée très étroite de leur cadre de vie. Le seul lieu qui leur importe vraiment, c'est la cour d'habitation construite sur la parcelle possédée. D'où le sentiment fréquent que l'appropriation symbolique de l'espace de vie n'est possible que lorsqu'on en est le propriétaire légal. On a pu observer que les locataires pauvres ou les épouses en situation matrimoniale précaire s'impliquaient peu dans l'assainissement ou l'aménagement de la cour. Les épouses ne s'approprient pas le lieu parce qu'elles sont « chez leur mari »!

28. À cet égard, Robert Vuarin (1993, « Recourir à la solidarité coutumière ? » in Joseph BunetJailly, Se soigner au Mali, Paris: Karthala-Orstom : 342 p.) a pu observer dans la périphérie urbaine de Bamako un déplacement général de la sociabilité intra-familiale vers la sociabilité extrafamiliale. Il semble bien que dans ces nouveaux espaces urbains, les réseaux des voisins, des amis, des collègues, des connaissances et des relations est en train de prendre le pas sur celui des parents.

29. Selon sa situation dans le plan de lotissement, le prix d'une parcelle d'une surface moyenne de $400 \mathrm{~m}^{2}$ varie de 500000 FCFA à 2000000 FCFA

30. Qui a pour caractéristique de confondre les domaines publics, communs et privés. 


\section{RÉSUMÉS}

L'article réexamine les principes de la propriété foncière en Afrique de l'Ouest, montrant en particulier que c'était le principe de transmission qui métamorphosait la propriété individuelle en propriété lignagère commune. Ces propriétés communes étaient garanties de l'intérieur par la menace de sanctions magico-religieuses qui ne constituaient une protection efficace contre les tentatives de vol que dans la mesure où la croyance dans leur efficacité était partagée. Aujourd'hui, dans les milieux populaires, c'est le respect du code partagé de l'honneur et de la honte qui garantit, mieux que le Droit, l'exécution réciproque des obligations contractuelles de l'échange. Mais, tandis que la marchandisation brutale des domaines ancestraux se traduit par une spéculation foncière intense et par la privatisation illégale de l'espace public, la prolifération normative favorise le droit du « plus riche » et accroît encore la gravité des conflits fonciers.

The article re-examines the principles of land tenure in West Africa, showing in particular that it was the principle of transfer that metamorphosed personal property into common family property. Commonly owned properties were effectively protected from theft by the threat of magic sanctions but only in as far the belief in their effectiveness was shared. Today, it is respect of the shared code of honour and shame, more than the law, that guarantees the reciprocal execution of contractual obligations of exchange. But while the violent commodification of ancestral fields is being translated into intense land speculation and the illegal privatization of public space, normative proliferation supports the right of the richest and continues to increase the severity of conflicts about land.

\section{AUTEUR}

\section{JACKY BOUJU}

Anthropologue, CEMAf-Université de Provencebouju@mmsh.univ-aix.fr 